\title{
Enzyme analysis of the polyketide synthase leads to the discovery of a novel analog of the antibiotic a-lipomycin
}

\author{
Satoshi Yuzawa ${ }^{1}$, Clara H Eng ${ }^{1,2,3}$, Leonard Katz ${ }^{1,3}$ and Jay D Keasling 1,2,3,4,5,6 \\ The Journal of Antibiotics (2014) 67, 199-201; doi:10.1038/ja.2013.110; published online 30 October 2013
}

Keywords: broad substrate specificity; isoleucine; lipomycin synthase

$\alpha$-Lipomycin is an acyclic polyene antibiotic isolated from Streptomyces aureofaciens Tü117 (Figure 1a). ${ }^{1}$ Although cyclic polyene antibiotics such as nystatin and amphotericin generally show activity against fungi, $\alpha$-lipomycin was reported to be a Grampositive bacteria-specific antibiotic. ${ }^{1}$ The biosynthetic gene cluster of $\alpha$-lipomycin was characterized by Bihlmaier et al. ${ }^{2}$ They revealed the presence of four polyketide synthase (PKS) genes, lipPks1-lipPks4, as well as genes that encode a nonribosomal peptide synthase, lipNrps, and a methyltransferase, lipMt, that together are responsible for the biosynthesis of $\beta$-lipomycin, the aglycone of $\alpha$-lipomycin.

Recently, we showed biochemically that LipPks1 uses isobutyrylCoA to catalyze the polyketide chain initiation reaction and methylmalonyl-CoA for a single elongation reaction in vitro. ${ }^{3}$ Interestingly, several other acyl-CoAs were also accepted as starter substrates to produce the corresponding diketide intermediates. ${ }^{3}$ The $k_{\text {cat }}$ and $K_{\mathrm{m}}$ values for 2-methylbutyryl-CoA were comparable to those of isobutyryl-CoA, whereas the other starters showed significantly lower $k_{\text {cat }} / K_{\mathrm{m}}$ values. $^{3}$ These in vitro kinetic parameters suggested that an analog of $\alpha$-lipomycin, where 2-methylbutyryl-CoA is used in the chain initiation reaction, could be produced if the intracellular concentration of 2-methylbutyryl-CoA was increased in S. aureofaciens Tü117. In this study, we grew S. aureofaciens Tü117 in HA medium ( $1 \%$ malt extract, $0.4 \%$ yeast extract, $0.4 \%$ glucose and $1 \mathrm{~mm} \mathrm{CaCl}_{2}$ ) in the presence of isoleucine, a well-known precursor of 2-methybutyryl-CoA. Here, we report a novel analog of $\alpha$-lipomycin that was isolated from the host using the above growth conditions. Structural analysis determined the compound to be 21-methyl- $\alpha$-lipomycin (Figure 1b), which confirmed our prediction. We also report the antibiotic activity of the analog against Bacillus subtilis, a representative Gram-positive bacterium.

S. aureofaciens Tü117 was cultured in 1 liter of HA medium containing $20 \mathrm{~mm}$ isoleucine. As a control, the strain was grown in 1 liter of HA medium in the absence of isoleucine. After 7 days, mycelia of each culture were extracted with acetone. After the extracts were concentrated, they were combined with their corresponding supernatants and acidified with $\mathrm{HCl}(\mathrm{pH}=\sim 4)$. The resulting aqueous solutions were then extracted with ethyl acetate and concentrated. The crude extracts were analyzed by LC-MS. As shown in Figure 2, we found that the culture grown in the presence of isoleucine exhibited a substantial amount of a compound that had a mass shift of +14 relative to $\alpha$-lipomycin compared with the culture grown in the absence of isoleucine. To confirm the structure, we purified the analog by HPLC, obtaining $10 \mathrm{mg}$ from 1 liter of the HA + isoleucine culture, a yield comparable to that of $\alpha$-lipomycin purified from 1 liter of HA culture ( $9 \mathrm{mg})$.

LC-MS analysis of $\alpha$-lipomycin showed the presence of two sets of intense signals at $m / z=588.4,589.4,590.4$ and 591.4 and at $m / z=458.3,459.3,460.3$ and 461.3 (Supplementary Figure 1A), which correspond to $[\mathrm{M}+\mathrm{H}]^{+}$of $\alpha$-lipomycin (theoretical exact mass $=588.31$ ) and $\beta$-lipomycin (theoretical exact mass $=458.25$ ), respectively. Because we observed a single peak in the LC, we concluded that the MS signals for $\beta$-lipomycin were the fragmented ions of $\alpha$-lipomycin. Analysis of the analog also showed the presence of two sets of intense signals at $m / z=602.4,603.4,604.4$ and 605.4 and at $m / z=472.3,473.3,474.3$ and 475.3 (Supplementary Figure 1B). The theoretical exact mass of $[\mathrm{M}+\mathrm{H}]^{+}$of 21 -methyl$\alpha$-lipomycin is 602.33. The fragmented ions indicate that both $\alpha$-lipomycin and the analog have the same sugar moiety, and that the +14 mass originates from a change in the backbone structure.

The orange-red color of $\alpha$-lipomycin stems from the conjugated double-bond structure. The UV spectrum of $\alpha$-lipomycin exhibited an absorbance at $448 \mathrm{~nm}$ with $\varepsilon_{448}=29800$ (Supplementary Figure 2, dotted line). As expected, the spectrum of the analog was nearly identical to that of $\alpha$-lipomycin, $\lambda_{\max }=442 \mathrm{~nm}$ with $\varepsilon_{442}=27600$

${ }^{1}$ QB3 Institute, University of California, Berkeley, CA, USA; ${ }^{2}$ Department of Chemical and Biomolecular Engineering, University of California, Berkeley, CA, USA; ${ }^{3}$ Synthetic Biology Engineering Research Center, Emeryville, CA, USA; ${ }^{4}$ Department of Bioengineering, University of California, Berkeley, CA, USA; ${ }^{5}$ Joint BioEnergy Institute, Emeryville, CA, USA and 6 Lawrence Berkeley National Laboratory, Physical Bioscience Division, Berkeley, CA, USA

Correspondence: Professor JD Keasling, Joint BioEnergy Institute, 5885 Hollis Street, Emeryville, CA 94608, USA.

E-mail: keasling@berkeley.edu

Received 21 June 2013; revised 26 September 2013; accepted 3 October 2013; published online 30 October 2013 
<smiles>CC(C=CC=CC=CC=CC(=O)C1=C(O)[C@@H](NCCC(=O)O)N(C)C1=O)=CC(=O)OC1C[C@@H](O)[C@@H](O)[C@H](C)O1</smiles><smiles>CCC(C)C(/C=C(\C)C=CC=CC=CC=CC(=O)C1=C(O)[C@@H](CCC(=O)O)N(C)C1=O)O[C@H]1C[C@@H](O)[C@@H](O)[C@H](C)O1</smiles>

21-Methyl- $\alpha$-lipomycin

Figure 1 Chemical structures of $\alpha$-lipomycin (a) and 21 -methyl- $\alpha$ lipomycin (b).

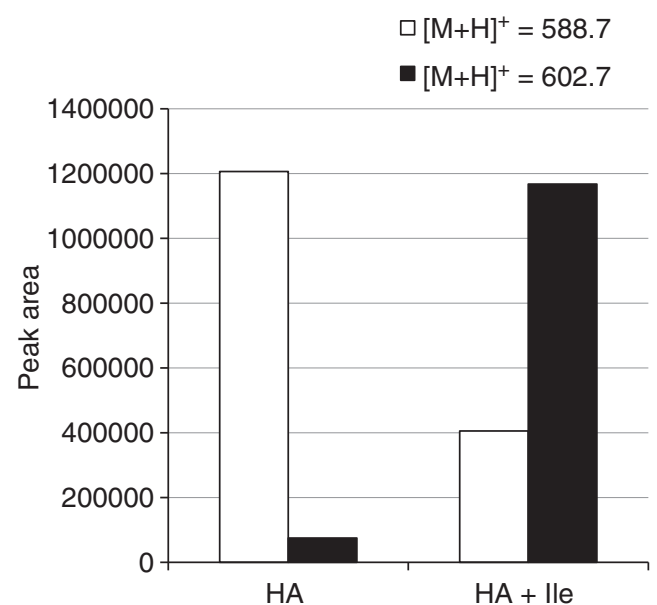

Figure 2 LC-MS analysis of the extract from $S$. aureofaciens Tü117. S. aureofaciens Tü 117 was grown in the $\mathrm{HA}$ medium for 7 days at $30^{\circ} \mathrm{C}$ in the absence or the presence of isoleucine. Production of $\alpha$-lipomycin $\left(\mathrm{m} / z=588.7[\mathrm{M}+\mathrm{H}]^{+}\right)$and the +14 analog $\left(\mathrm{m} / z=602.7[\mathrm{M}+\mathrm{H}]^{+}\right)$was analyzed by LC-MS.

(Supplementary Figure 2, solid line). These data indicate that the analog has a very similar $\pi$-electron conjugation system to $\alpha$-lipomycin.

In order to elucidate the structure of the analog, we performed NMR analysis for both $\alpha$-lipomycin and the analog. The ${ }^{1} \mathrm{H}$ NMR spectra of the analog and $\alpha$-lipomycin were nearly identical except for the two regions: $\delta_{\mathrm{H}} 0.88-1.02$ p.p.m. and $\delta_{\mathrm{H}} 1.54-1.83$ p.p.m. (Table 1 and Supplementary Figures $3 \mathrm{~A}, 7 \mathrm{~A}$, and 11). These proton signals were assigned to be $\mathrm{H} 20, \mathrm{H} 21$ and $\mathrm{H} 25$ for $\alpha$-lipomycin and H20, $\mathrm{H} 21, \mathrm{H} 25$ and the protons on the methyl group at C21 for the analog, respectively, by analysis of the 2D NMR spectra, DQF-COSY, TOCSY and HSQC (Supplementary Figures 4-6 and 8-10). It should be noted that the proton signal for $\mathrm{H} 21$ in the analog is a multiplet at $\delta_{\mathrm{H}}$ 1.68 p.p.m., whereas the corresponding proton in $\alpha$-lipomycin is a
Table $1{ }^{1} \mathrm{H}$ and ${ }^{13} \mathrm{C}$ NMR chemical shifts of $\alpha$-lipomycin and 21-methyl- $\alpha$-lipomycin in methanol- $d_{4}$

\begin{tabular}{|c|c|c|c|c|}
\hline \multirow[b]{2}{*}{ Position } & \multicolumn{2}{|c|}{$\alpha$-Lipomycin } & \multicolumn{2}{|c|}{ 21-Methyl-a-lipomycin } \\
\hline & $\delta_{H}$ & $\delta_{C}$ & $\delta_{H}$ & $\delta_{C}$ \\
\hline \multirow[t]{2}{*}{2} & $2.14(\mathrm{~m}, 1 \mathrm{H})$ & 25.05 & $2.13(\mathrm{~m}, 1 \mathrm{H})$ & 24.98 \\
\hline & $2.26(\mathrm{~m}, 1 \mathrm{H})$ & & $2.25(\mathrm{~m}, 1 \mathrm{H})$ & \\
\hline 3 & $2.26(\mathrm{~m}, 2 \mathrm{H})$ & 29.02 & $2.27(\mathrm{~m}, 2 \mathrm{H})$ & 29.04 \\
\hline 4 & $3.90(\mathrm{t}, 1 \mathrm{H})$ & 66.24 & $3.87(\mathrm{t}, 1 \mathrm{H})$ & 66.39 \\
\hline $8-15$ & $6.26-8.11(8 \mathrm{H})$ & 121.73-145.97 & $6.25-8.09(8 \mathrm{H})$ & $122.25-145.67$ \\
\hline 17 & $5.71(\mathrm{~d}, 1 \mathrm{H})$ & 139.88 & $5.72(\mathrm{~d}, 1 \mathrm{H})$ & 139.70 \\
\hline 18 & $2.87(\mathrm{~m}, 1 \mathrm{H})$ & 37.47 & $2.88(\mathrm{~m}, 1 \mathrm{H})$ & 37.09 \\
\hline 19 & $3.33(\mathrm{dd}, 1 \mathrm{H})$ & 88.63 & $3.42(\mathrm{dd}, 1 \mathrm{H})$ & 88.69 \\
\hline 20 & $1.79(\mathrm{~m}, 1 \mathrm{H})$ & 32.21 & $1.55(\mathrm{~m}, 1 \mathrm{H})$ & 39.33 \\
\hline 21 & $0.99(\mathrm{~d}, 3 \mathrm{H})$ & 20.41 & $1.68(\mathrm{~m}, 2 \mathrm{H})$ & 25.91 \\
\hline 23 & $1.86(\mathrm{~s}, 3 \mathrm{H})$ & 12.79 & $1.85(\mathrm{~s}, 3 \mathrm{H})$ & 12.75 \\
\hline 24 & $1.05(\mathrm{~d}, 3 \mathrm{H})$ & 18.62 & $1.07(\mathrm{~d}, 3 \mathrm{H})$ & 18.90 \\
\hline 25 & $0.96(d, 3 H)$ & 18.80 & $0.91(\mathrm{~d}, 3 \mathrm{H})$ & 16.26 \\
\hline $\mathrm{H}_{3} \mathrm{C}$ & - & - & $0.92(t, 3 \mathrm{H})$ & 11.97 \\
\hline $1^{\prime}$ & $4.87(\mathrm{t}, 1 \mathrm{H})$ & 100.42 & $4.87(\mathrm{t}, 1 \mathrm{H})$ & 100.62 \\
\hline \multirow[t]{2}{*}{$2^{\prime}$} & $1.65(\mathrm{~m}, 1 \mathrm{H})$ & 39.57 & $1.65(\mathrm{~m}, 1 \mathrm{H})$ & 39.56 \\
\hline & $2.05(\mathrm{~m}, 1 \mathrm{H})$ & & $2.05(\mathrm{~m}, 1 \mathrm{H})$ & \\
\hline $3^{\prime}$ & $4.03(\mathrm{dt}, 1 \mathrm{H})$ & 69.39 & $4.03(\mathrm{dt}, 1 \mathrm{H})$ & 69.39 \\
\hline $4^{\prime}$ & $3.18(\mathrm{dd}, 1 \mathrm{H})$ & 74.50 & $3.18(\mathrm{dd}, 1 \mathrm{H})$ & 74.48 \\
\hline $5^{\prime}$ & $3.71(\mathrm{~m}, 1 \mathrm{H})$ & 70.59 & $3.73(\mathrm{~m}, 1 \mathrm{H})$ & 70.61 \\
\hline $6^{\prime}$ & $1.27(\mathrm{~d}, 3 \mathrm{H})$ & 18.62 & $1.26(\mathrm{~d}, 3 \mathrm{H})$ & 18.62 \\
\hline
\end{tabular}

doublet at $\delta_{\mathrm{H}} 0.99$ p.p.m. In addition, a new proton signal appeared at 0.92 p.p.m. in the analog. On the basis of these NMR spectra, in conjunction with the MS and UV data, we determined the analog structure to be 21 -methyl- $\alpha$-lipomycin.

We also determined the MIC values for both compounds against B. subtilis and Escherichia coli, the latter a representative Gramnegative bacterium. The potencies were the same for the two compounds: MIC for B. subtilis $=0.78 \mu \mathrm{g} \mathrm{ml}^{-1}$ and MIC for E. coli $>200 \mu \mathrm{g} \mathrm{ml}^{-1}$. The results indicate that the activity is not affected by the structure of the starter substrate.

The avermectin family of polyketides is produced, in Streptomyces avermitilis, by a single PKS system whose loading didomain is capable of using isobutyryl-CoA or 2-methylbutyryl-CoA to start the synthesis. ${ }^{4,5}$ Because only a single lipomycin congener was isolated from S. aureofaciens Tü117,1,2,6,7 it was assumed that the LipPks1 loading didomain was specific for isobutyryl-CoA.

Intrinsic substrate specificity of an enzyme can only be determined by in vitro kinetic analysis. Our previous kinetic studies of LipPks1 revealed that the $k_{\text {cat }} / K_{\mathrm{m}}$ values were comparable between isobutyryl$\mathrm{CoA}$ and 2-methylbutyryl-CoA, ${ }^{3}$ suggesting that the lipomycin synthase could produce a methylated analog of $\alpha$-lipomycin. In this work, we demonstrated the production of 21-methyl- $\alpha$-lipomycin by increasing the intracellular concentration of 2-methylbutyryl-CoA. This highlights the importance of determining in vitro kinetic parameters for loading didomains of PKSs to expand the variety of identifiable drug candidates and thereby accelerate drug discovery.

\section{CONFLICT OF INTEREST}

JDK has a financial interest in Amyris, LS9 and Lygos. The other authors declare no competing financial interest. 


\section{ACKNOWLEDGEMENTS}

This work was funded by the Advanced Research Projects Agency-Energy (ARPA-E), U.S. Department of Energy, under award number DE-AR0000091, by the National Science Foundation, award No. EEC-0540879 to the Synthetic Biology Research Center and by the Joint BioEnergy Institute (JBEI), which is funded by the U.S. Department of Energy, Office of Science, Office of Biological and Environmental Research, under contract number DE-AC0205CH11231. We thank Andreas Bechthold for providing us S. aureofaciens Tü117 and Jeffrey Pelton for assistance with NMR analysis.

1 Kunze, B., Schabach., K, Zeeck, A. \& Zahner, H. Metabolic products of microorganisms.3. Lipomycins.1. Isolation, characterization and first investigations of structure and mechanism of action. Archiv. Fur. Mikrobiologie 86, 147-174 (1972).

2 Bihlmaier, C. et al. Biosynthetic gene cluster for the polyenoyltetramic acid alphalipomycin. Antimicrob. Agents Chemother. 50, 2113-2121 (2006).

3 Yuzawa, S., Eng, C. H., Katz, L. \& Keasling, J. D. Broad substrate specificity of the loading didomain of the lipomycin polyketide synthase. Biochemistry 52, 3791-3793 (2013).

4 Burg, R. W. et al. Avermectins, new family of potent anthelmintic agents: producing organism and fermentation. Antimicrob. Agents Chemother. 15, 361-367 (1979).

5 Ikeda, H., Nonomiya, T., Usami, M., Ohta, T. \& Omura, S. Organization of the biosynthetic gene cluster for the polyketide anthelmintic macrolide avermectin in Streptomyces avermitilis. Proc. Natl Acad. Sci. USA 96, 9509-9514 (1999).

6 Schabach., K \& Zeeck, A. Lipomycins.2. Composition of alpha and beta lipomycin. Tetrahedron Lett. 14, 2691-2694 (1973).

7 Zeeck, A. Lipomycins.3. Isolation and identification of methyl 2,6-dideoxy-d-ribohexosides. Liebigs Ann. Chem. 1975, 2079-2088 (1975).

Supplementary Information accompanies the paper on The Journal of Antibiotics website (http://www.nature.com/ja) 